\title{
Análisis de los factores que tienen influencia en la disminución de resistencias residuales por disolución alcalina en los morteros intersticiales de hormigones con cemento aluminoso. Evaluación de los límites de riesgo en el caso de los techos
}

\author{
Analysis of the factors which have an influence on the reduction \\ of residuary strengths due to alkaline solution in the interstitial \\ mortars of concretes wich aluminous cement. Assessment of the \\ risk limits in the case of roof beams
}

\author{
FERRAN GOMÀ GINESTÀ, Dr. En Ciències Químiques \\ Prof. Titular Univ. Politècnica de Catalunya. Laboratori de Recerca Química. Dep. de Construccions Arquitectòniques I
}

ESPAÑA

Fecha de recepción: 17-VI-95

RESUMEN

Las escasas referencias que existen en la bibliografia sobre este tema, y su identificación sobre los factores que tienen influencia sobre la disminución de resistencias en el hormigón de cemento aluminoso por efecto de la alcalinidad, no ha permitido aún ver claramente los limites que pueden dar lugar a una situación de riesgo en los elementos estructurales por esta causa.

Se presenta un estudio que trata de medir la influencia cuantitativa de diferentes parámetros, con la misma o semejante magnitud que se presentan en los tipos usuales de hormigones de vigas existentes con cemento aluminoso propio de nuestro mercado.

Los parámetros ensayados han sido: la influencia de la concentración de iones (OH)- libres, la clase de ión alcalino, la temperatura, el tiempo, la presencia o ausencia de $\mathrm{CO}_{2}$, el grado de conversión, los parámetros físicos, porosidades, densidades, etc., $y$ han sido determinadas las fases existentes por XRD, DTA y TG, antes y después de la acción alcalina.

De los resultados obtenidos en múltiples series de morteros Rilem y micro-morteros, se puede ver la posibilidad de establecer el entorno de parámetros que definen zonas sin riesgo.

\section{SUMMARY}

The scarce references which exist in the bibliography on this subject and their vagueness on the factors which have an influence on the reduction of the strength of aluminous cement concrete due to the effect of alkalinity, have not yet allowed the limits to be clearly seen which could give rise to a situation of risk in the structural elements due to this cause.

A study is presented which endeavours to measure the quantitative influence of different parameters of the same or of a similar conditions to those presented in the usual types of concrete in existing beams with aluminous cement characteristic of our market.

The parameters tested were: the influence of the concentration of free ions $\mathrm{OH}$, the class of alkaline ion, the temperature, the time, the presence or absence of $\mathrm{CO}_{2}$, the degree of conversion, the physical parameters, porosities, densities, etc., and the phases existing were determined by $X R D, D T A$ and $T G$ before and after the alkaline action.

From the results obtained in numerous series of Rilem mortars and micro-mortars, it is possible to see the possibility of establishing the setting of parameters which define areas without risk.
PALABRAS CLAVE: Disgregación; Hidrólisis Alcalina; Cemento Aluminoso; Resistencias; Conversión.
KEYWORDS: Disgregation; Alkaline Hydrolysis; Aluminous Concrete; Strengths; Conversion. 


\section{INTRODUCCIÓN}

La bibliografia sobre este tema se reduce a referencias sobre la hipótesis implícita de su existencia, más que a medidas cuantitativas sobre su grado y los efectos que pueden tener sobre las resistencias de los hormigones (1) (2).

Un trabajo específico, sobre la clarificación de las alteraciones de hormigones de cemento aluminoso por posible disgregación alcalina, es el RENGADE (3) del año 1936. El trabajo examina las concentraciones de alúmina y calcio que pasan a las disoluciones alcalinas de contacto de muestras pulverizadas con diferentes concentraciones de iones $\mathrm{OH}^{-}$a la temperatura ambiental. Pone en evidencia el paso de los álcalis contenidos en los áridos en la fase líquida de los hormigones estudiados en función de la temperatura y la concentración de hidróxido de calcio, y determina, de forma cualitativa, la agresión sobre series de hormigones sometidos con otros tipos de cementos con áridos portadores de álcalis que especifica con unas arenas con un $5,2 \%$ de álcalis.

No obstante, no determina cuantitativamente el efecto sobre las resistencias en los hormigones de cemento aluminoso ni, mucho menos, discrimina otros efectos diferentes de la propia disolución alcalina, como son el grado de conversión, las reacciones citadas "álcali-árido", o bien, la de "álcali-carbonato". Tampoco hace un estudio de las fases de nueva formación que se producen y como afectan al desarrollo de las resistencias.

Estos puntos son, según nuestro criterio, los objetivos más coherentes con tal de aproximarnos en la medida de lo posible, a poder establecer, al menos, que es lo que hay del efecto neto de la disgregación alcalina sobre las resistencias, y cuales serían los límites del entorno entre las zonas de riesgo y las de seguridad, en función de los parámetros que pueden tener influencia en la disolución alcalina con efecto sobre las resistencias residuales.

\section{VISIÓN DE LA INVESTIGACIÓN}

La investigación se realiza con cemento aluminoso actual de España. Se compone de las siguientes etapas:

-Ensayo con pasta pura, con una relación agua/cemento 0,35 , curada en inmersión durante tres meses a temperatura ambiental inferior a $20^{\circ} \mathrm{C}$ y después conservada en seco, reducida a tamaño inferior a $88 \mu \mathrm{m}$, a temperatura ambiental.

-Tratamiento en reactor a presión normal, a diferentes temperaturas hasta $80^{\circ} \mathrm{C}$, en soluciones de $\mathrm{NaOH}$ y $\mathrm{K}_{2} \mathrm{CO}_{3}$ a diferentes concentraciones hasta $2 \mathrm{M}$.

\section{INTRODUCTION}

The bibliography on this subject is reduced to references on the implicit hypothesis of its existence, rather than to quantitative measurements of its degree and the effects which it can have on the strengths of concretes (1) (2).

A work specifically on the clarification of the alterations to aluminous cement concretes due to possible alkaline disgregation is that of RENGADE (3) from 1936. The work examines the concentrations of alumina and calcium which pass into the contact alkaline solutions of ground samples with different concentrations of $\mathrm{OH}^{-}$ions at ambient temperature. It demonstrates the passage of the alkalis contained in the aggregates in the liquid phase of the concretes studied depending on the temperature and the concentration of calcium hydroxide, and determines in a qualitative manner the aggression on series of concretes subjected with other types of cements to alkali holding aggregates which it specifies with sand with $5.2 \%$ of alkalis.

However, it does not quantitatively determine the effect on the strengths in aluminous cement concretes nor, much less, does it differentiate between other different effects of the alkaline solution itself, such as the degree of conversion, the so-called "alkali-aggregate" or the "alkali-carbonate" reactions. Nor does it study the newly formed phases which are produced and how they affect the development of the strengths.

These points are, in our opinion, the most coherent objectives in order to come as close as possible to being able to establish at least the extent of the net effect of the alkaline disgregation on the strengths, and what the limits of the environment would be between areas of risk and those of safety, depending on the parameters which may have an influence on the alkaline solution with an effect on the residuary strengths.

\section{OVERVIEW OF THE RESEARCH}

The research was carried out with current aluminous cement from Spain. It was composed of the following stages:

-Test with neat aluminous cement paste with a water/cement ratio 0.35 cured by immersion for three months at room temperature below $20^{\circ} \mathrm{C}$ and then maintained dry, and reduced to a size below $88 \mu \mathrm{m}$ at room temperature.

-Treatment in a reactor at normal pressure at different temperatures up to $80^{\circ} \mathrm{C}$ in solutions of $\mathrm{NaOH}$ and $\mathrm{K}_{2} \mathrm{CO}_{3}$, at different concentrations up to $2 \mathrm{M}$. 
-Ensayo de morteros con microprobetas $50 \times 15 \times 15 \mathrm{~mm}$, y $40 \times 40 \times 160 \mathrm{~mm}$.

Con morteros previamente curados durante un año, se han hecho series control-blanco, y se han comparado con series sometidas a tratamientos en las mismas condiciones que las de las pastas puras. Series en paralelo han permitido diferenciar el efecto de la conversión del de la acción alcalina sobre las resistencias.

Se han seguido las variaciones de los parámetros físicos y químicos en función de los tratamientos y se ha obtenido información por las técnicas de análisis químico C.A., A.T.D., T.G. y X.R.D.

\section{COMPOSICIÓN DE MORTEROS}

\subsection{Materiales}

Cemento: Cemento aluminoso del mercado de España, fabricado en 1992. Características que se exponen en la Tabla I.

Áridos: Arenas de Cuarzo, prácticamente sin feldespatos, en dos fracciones granulométricas para cada tipo de mortero. En los morteros Rilem hemos utilizado, además, un conjunto calcáreo en series alternativas.

\subsection{Proporciones y Parámetros de los Morteros}

Con el fin de poder conseguir una información válida para las aplicaciones técnicas de resultados, hemos establecido dos tipos de series con diferente dosificación de cemento, una alrededor de $250 \mathrm{~kg} / \mathrm{m}^{3}$, y otra sobre los $450 \mathrm{~kg} / \mathrm{m}^{3}$, con $0,6-0,7$ y $0,35-0,40$ de relación agua/cemento, respectivamente, de acuerdo con las utilizadas en la prefabricación de vigas para techos. Esto se ha hecho a partir de los análisis encontrados en edificios en los que hemos realizado dictámenes analíticos para su rehabilitación arquitectónica. De forma análoga, hemos hecho series con diferentes porosidades siguiendo el mismo criterio de los datos de las vigas reales. Tabla II.

Hemos preparado pasta pura de cemento aluminoso por hidratación en las condiciones normales de temperatura ambiental, con una relación de agua/cemento de 0,35 . Han estado curadas en inmersión a temperatura inferior a $20^{\circ} \mathrm{C}$ y después mantenidas en una atmósfera de $45 \%$ de humedad relativa en un desecador. Los valores de su composición química centesimal se exponen en la Tabla I.
-Mortar test with microsamples 50x15x15 mm and $40 \times 40 \times 160 \mathrm{~mm}$.

A series of blank controls were carried out with RILEM mortars previously cured for one year at temperature between 8 and $12^{\circ} \mathrm{C}$, and these were compared with series subjected to treatments in the same conditions as those of the neat pastes. Parallel series made it possible to differentiate the effect of the conversion of the alkaline action on the strengths.

The variations of the physical and chemical parameters were monitored, depending on the treatments, and information was obtained by the chemical analysis techniques C.A., D.T.A., G.T. and X.R.D.

\section{COMPOSITION OF MORTARS}

\subsection{Materials}

Cement:Aluminous cement from the Spanish market manufactured in 1992. Characteristics given in Table I.

Aggregates:Quartz sand, practically without feldspars, in two granulometric fractions for each kind of mortar. With the Rilem mortars we moreover used fine aggregates of calcareous materials in alternate series.

\subsection{Proportions and Parameters of the Mortars}

In order to be able to obtain valid information for the technical applications of results, we established two types of series with a different dosage of cement, one around $250 \mathrm{~kg} / \mathrm{m}^{3}$, and the other around $450 \mathrm{~kg} / \mathrm{m}^{3}$, with a water/cement ratio of $0.6-0.7$ and $0.35-0.40$ respectively, in accordance with those used in the prefabrication of beams for roofs. This was carried out starting from the analyses found in buildings in which we undertook analytical reports for their architectural restoration. In an analogous manner, we created series with different porosities, following the same criterion, starting from the data on the actual beams. Table II.

We prepared neat aluminous cement paste by hydration in normal conditions at room temperature, with a water/cement ratio of 0.35 . It was cured by immersion at a temperature below $20^{\circ} \mathrm{C}$ and then maintained in an atmosphere with $45 \%$ relative humidity in a drier. The values of its centesimal chemical composition are given in Table I. 
TABLA I (TABLE I)

Composición del cemento aluminoso y de la pasta pura hidratada (Chemical analysis of aluminous cement and neat hydrated paste)

\begin{tabular}{|c|c|c|}
\hline \multicolumn{3}{|c|}{ Análisis químicos $(\%)$ / Values in (\%) } \\
\hline & $\begin{array}{l}\text { Cemento } \\
\text { Aluminoso } \\
\text { (Aluminous } \\
\text { Cement) }\end{array}$ & $\begin{array}{l}\text { Pasta Pura Hidratada } \\
\text { A/C 0,35 Edad } 1 \text { año } \\
\text { (Neat Hydrated Paste } \\
\text { A/C 0,35 AGE } 1 \text { year) }\end{array}$ \\
\hline $\mathrm{Si} \mathrm{O}_{2}$ & 3,0 & 2,3 \\
\hline $\mathrm{Al}_{2} \mathrm{O}_{3}$ & 39,7 & 30,1 \\
\hline $\mathrm{FeO}$ & 0,95 & -- \\
\hline $\mathrm{Fe}_{2} \mathrm{O}_{3}$ & 16,8 & 13,3 \\
\hline $\mathrm{CaO}$ & 36,8 & 27,4 \\
\hline $\mathrm{MgO}$ & 0,6 & 0,5 \\
\hline $\mathrm{SO}_{3}$ & 0,1 & 0,1 \\
\hline $\mathrm{Na}_{2} \mathrm{O}$ & 0,03 & 0,02 \\
\hline $\mathrm{K}_{2} \mathrm{O}$ & 0,04 & 0,03 \\
\hline $\mathrm{TiO}_{2}$ & 1,9 & 1,5 \\
\hline $\begin{array}{l}\text { Pérdida por calcinación } \\
\text { (Loss on ignition) }\end{array}$ & 0,35 & 24,5 \\
\hline
\end{tabular}

TABLA || (TABLE II)

Parámetros físicos de los morteros preparados $\left({ }^{*}\right)$ (Physical properties of morters) (*)

\begin{tabular}{|c|c|c|c|c|}
\hline & $\begin{array}{c}\text { Densidad (*) } \\
\text { másica } \\
\left.(B u / k \text { Density) })^{*}\right) \\
\left(\mathrm{g} / \mathrm{cm}^{3}\right)\end{array}$ & $\begin{array}{c}\text { Densidad (*) } \\
\text { aparente } \\
\text { (Apparent } \\
\text { Density)/ } \\
\text { g/cm } \\
\end{array}$ & $\begin{array}{c}\text { Capacidad } \\
\text { absorción agua } \\
\text { (Water } \\
\text { absorption) } \\
\%\end{array}$ & $\begin{array}{c}\text { Porosidad } \\
\text { total vol. } \\
\text { (Volume of } \\
\text { permeable } \\
\text { pore space) } \\
\%\end{array}$ \\
\hline $\begin{array}{l}\text { Series: } \\
\text { A,D,E,H,I. } \\
\text { Xi }\end{array}$ & 1,72 & 2,31 & 13,5 & 23,8 \\
\hline $\begin{array}{l}\text { Series: } \\
\mathbf{B}, \mathbf{C}, \mathbf{F}, \mathbf{G}, \mathbf{K} \text {. } \\
\text { Xi }\end{array}$ & 2,00 & 2,37 & 7,8 & 15,6 \\
\hline $\begin{array}{l}\sigma \\
\text { C.V.\% } \\
\end{array}$ & $\begin{array}{l}0,04 \\
2,03 \\
\end{array}$ & $\begin{array}{l}0,08 \\
3,57 \\
\end{array}$ & $\begin{array}{c}0,77 \\
8,4 \\
\end{array}$ & $\begin{array}{l}1,54 \\
8,34 \\
\end{array}$ \\
\hline $\begin{array}{l}\text { Series: } \\
\text { L,M,N,O,P,Q. } \\
\text { Xi } \\
\sigma \\
\text { C.V.\% }\end{array}$ & $\begin{array}{l}2,26 \\
0,03 \\
1,32\end{array}$ & $\begin{array}{l}2,68 \\
0,02 \\
0,80\end{array}$ & $\begin{array}{l}6,8 \\
0,73 \\
10,7\end{array}$ & $\begin{array}{c}15,0 \\
0,82 \\
5,4\end{array}$ \\
\hline
\end{tabular}

(*) Los valores corresponden a los de la norma ASTM "Bulck and Apparent Densities"/ ASTM values. 


\section{PREPARACIÓN Y CURAdO DE LAS MUESTRAS}

Los morteros se han preparado con amasadora planetaria y compactada, por el ensayo Rilem, de acuerdo con la normativa UNE. Por lo que se refiere a los micromorteros, ha resultado más eficiente compactar con micro-vibradora. Después del curado previo en cámara durante 24 horas, se ha curado en inmersión a temperatura siempre inferior a $\operatorname{los} 10^{\circ} \mathrm{C}$, durante un año previo a los ensayos de roturas.

\section{RESULTADOS EXPERIMENTALES}

\subsection{Composición Química de Óxidos}

Se ha determinado la composición química de óxidos en la pasta pura hidratada, curada en inmersión durante 90 días y mantenida en equilibrio con la atmósfera durante un año. Los resultados pueden verse en la Tabla I. Se ha determinado la composición de las pastas atacadas por soluciones alcalinas de diferente molaridad, con y sin carbonato. Los resultados se incluyen en la Tabla III.

Los procedimientos de los análisis químicos han sido los métodos gravimétricos (4), (5) y complexométricos (6) puestos a punto en trabajos anteriores y publicados en las referencias dadas. Los métodos complexométricos referenciales, son equivalentes a los normativos actuales propuestos por el CEN. (7).

Se ha determinado la composición de las disoluciones alcalinas de ataque sobre la pasta pura reducida a tamaño de partícula inferior a $88 \mu \mathrm{m}$, filtradas después de los tratamientos a diferentes temperaturas (Tabla III).

La evolución de las composiciones de los residuos insolubles, en función del tipo de ataque alcalino, se ha estudiado sobre los valores absolutos centrados en la suma de los óxidos mayoritarios de las respectivas composiciones en $\mathrm{Al}_{2} \mathrm{O}_{3}, \mathrm{CaO}$ y $\mathrm{Fe}_{2} \mathrm{O}_{3}$ (total), previamente totalizados al $100 \%$. Dichos valores se exponen en el diagrama de la Fig. 1.

\subsection{Ensayo a Compresión}

5.2.1.Micro-mortero: La preparación de estos morteros curados en inmersiones hasta la ruptura, se han ensayado a la edad de 120 días distribuidos en las siguientes series:

\section{PREPARATION AND CURING OF THE SAMPLES}

The mortars were prepared according to the UNE standard for the Rilem test. As far as the micro-mortars were concerned, the best results were obtained using a micro-vibration machine. After prior curing in a chamber for 24 hours, they were cured by immersion maintained at temperatura between 8 and $10^{\circ} \mathrm{C}$ for one year prior to the rupture tests.

\section{EXPERIMENTAL RESULTS}

\subsection{Chemical Composition of Oxides}

The chemical composition of oxides in the neat hydrated paste, cured by immersion for 90 days and maintained in equilibrium with the atmosphere at room temperature for one year was determined. The results are shown in Table I. The composition of the pastes attacked by alkaline solutions of different molarity, with and without carbonate, was determined. The results are included in Table III.

The procedures for the chemical analyses were gravimetric (4), (5) and complexometric (6) methods, established in previous works and published in the references given. The complexometric methods referred to are equivalent to the current standards proposed by the CEN (7).

The composition of the alkaline solutions attacking the neat paste reduced to a particle size of less than $88 \mu \mathrm{m}$ $\mu m$, filtered after the treatments at different temperatures, was determined. Table III.

The evolution of the compositions of the insoluble residues, depending on the type of alkaline attack, was studied, over the absolute values centred on the sum of the majority oxides of the respective compositions in $\mathrm{Al}_{2} \mathrm{O}_{3} \mathrm{CaO}$ and $\mathrm{Fe}_{2} \mathrm{O}_{3}$ (total) previously totalized to $100 \%$ These values are shown in the ternary diagram of Fig. 1 .

\subsection{Compression Test}

5.2.1.Micro-mortar: The preparation of these mortars, cured by immersion until rupture, weretested at an age of 120 days distributed in the following series: 
TABLA III (TABLE III)

Composición química de las pastas puras atacadas por disoluciones alcalinas (Chemical analysis of the neat pastes attacked by alkaline solutions)

Análisis químicos (\%) / Chemical analysis (\%)

\begin{tabular}{|c|c|c|c|c|c|c|c|}
\hline $\begin{array}{l}7 \text { Dias/Days } \\
\mathrm{K}_{2} \mathrm{CO}_{3} 2 \mathrm{M} \\
80^{\circ} \mathrm{C} \\
(*) 1\end{array}$ & & $\begin{array}{c}7 \\
\mathrm{NaOH} 2 \mathrm{M} \\
80^{\circ} \mathrm{C} \\
2\end{array}$ & $\begin{array}{c}30 \\
\mathrm{~K}_{2} \mathrm{CO}_{3} 2 \mathrm{M} \\
80^{\circ} \mathrm{C} \\
3\end{array}$ & $\begin{array}{c}30 \\
\mathrm{NaOH} 2 \mathrm{M} \\
80^{\circ} \mathrm{C} \\
4\end{array}$ & $\begin{array}{c}30 \\
\mathrm{~K}_{2} \mathrm{CO}_{3} 2 \mathrm{M} \\
60^{\circ} \mathrm{C} \\
5\end{array}$ & $\begin{array}{c}30 \\
\mathrm{~K}_{2} \mathrm{CO}_{3} 2 \mathrm{M} \\
20^{\circ} \mathrm{C} \\
6\end{array}$ & $\begin{array}{c}150 \\
\mathrm{~K}_{2} \mathrm{CO}_{3} 2 \mathrm{M} \\
80^{\circ} \mathrm{C} \\
7\end{array}$ \\
\hline $\begin{array}{ll}\mathrm{SiO}_{2} & \\
\mathrm{Al}_{2} \mathrm{O}_{3} & 2 \\
\mathrm{Fe}_{2} \mathrm{O}_{3} & 1 \\
\mathrm{CaO} & 2 \\
\mathrm{MgO} & \\
\mathrm{SO}_{3} \\
\mathrm{TiO}_{2} \\
\mathrm{Pérdida} \mathrm{por} \mathrm{Calcinación:}_{\text {(Loss on ignition): }} \\
20-950^{\circ} \mathrm{C}\end{array}$ & $\begin{array}{r}2,5 \\
22,9 \\
12,8 \\
23,9 \\
1,4 \\
0,1 \\
1,5 \\
n: \\
35,7\end{array}$ & $\begin{array}{c}2,3 \\
16,0 \\
15,8 \\
31,2 \\
1,6 \\
0,1 \\
1,6 \\
\\
\\
23,3\end{array}$ & $\begin{array}{l}2,6 \\
20,4 \\
14,9 \\
29,0 \\
1,6 \\
0,1 \\
1,4 \\
\\
\\
29,9\end{array}$ & $\begin{array}{c}2,6 \\
20,1 \\
18,7 \\
34,4 \\
1,5 \\
0,1 \\
1,5 \\
\\
\\
21,0\end{array}$ & $\begin{array}{c}2,6 \\
24,0 \\
12,4 \\
27,2 \\
1,4 \\
0,1 \\
1,5 \\
\\
\\
30,6\end{array}$ & $\begin{array}{c}2,4 \\
20,3 \\
12,5 \\
24,5 \\
1,5 \\
0,1 \\
1,6 \\
\\
\\
37,1\end{array}$ & $\begin{array}{c}2,4 \\
13,2 \\
15,9 \\
31,3 \\
1,5 \\
0,1 \\
1,5 \\
\\
\\
33,8\end{array}$ \\
\hline $\begin{array}{l}20-210^{\circ} \mathrm{C} \\
210-550^{\circ} \mathrm{C} \\
550-950^{\circ} \mathrm{C}\end{array}$ & $\begin{array}{r}5,6 \\
4,6 \\
25,5\end{array}$ & $\begin{array}{c}2,7 \\
13,0 \\
7,6\end{array}$ & $\begin{array}{c}4,7 \\
3,8 \\
21,2\end{array}$ & $\begin{array}{c}3,9 \\
12,4 \\
4,7\end{array}$ & $\begin{array}{c}7,5 \\
9,5 \\
13,6\end{array}$ & $\begin{array}{c}9,2 \\
10,6 \\
17,3\end{array}$ & $\begin{array}{c}2,9 \\
12,7 \\
18,2\end{array}$ \\
\hline $\begin{array}{l}\text { Fracción Soluble \% } \\
\text { (Soluble Fraction \%) } \\
\text { Residuo Insoluble \% } \\
\text { (Insoluble Residue \%) } 8\end{array}$ & $\begin{array}{l}16,9 \\
83,1\end{array}$ & $\begin{array}{l}26,7 \\
73,3\end{array}$ & $\begin{array}{l}16,0 \\
84,0\end{array}$ & $\begin{array}{l}30,0 \\
70,0\end{array}$ & $\begin{array}{l}15,5 \\
84,5\end{array}$ & $\begin{array}{l}17,6 \\
82,4\end{array}$ & $\begin{array}{l}14,5 \\
85,5\end{array}$ \\
\hline $\begin{array}{l}\mathrm{Al}^{(\mathrm{OH})_{4}}{ }^{-} \\
\text {Soluble }\end{array}$ & $\begin{array}{r}g / L \\
17,7\end{array}$ & 13,5 & 18,4 & 17,1 & 16,3 & 18,6 & 15,3 \\
\hline
\end{tabular}

(*) Posición de composiciones en el diagrama ternario de la Fig. 1

(Composition points on ternary diagram in Fig. 1)

\section{Efecto debido a conversión:}

Serie A: Control de baja dosificación.

Serie B: Control de alta dosificación.

Temperatura de curado siempre inferior a $+10^{\circ} \mathrm{C}$ (cámara frigorírica) y en inmersión.

Serie C: Alta dosificación con tratamiento únicamente térmico a $80^{\circ} \mathrm{C} 30$ días.

Serie D: Baja dosificación con tratamiento únicamente térmico a $80^{\circ} \mathrm{C} 30$ días.

Los resultados están expuestos en la Tabla IV.

\section{Effect due to conversion}

Series A:Control of low dosage

Series B:Control of high dosage

Curing temperature always below $+10^{\circ} \mathrm{C}$

(refrigeration chamber) and by immersion.

Series C:High dosage with solely heat treatment at $80^{\circ} \mathrm{C} 30$ days

Series D: Low dosage with solely heat treatment at $80^{\circ} \mathrm{C} 30$ days

The results are shown in Table IV. 


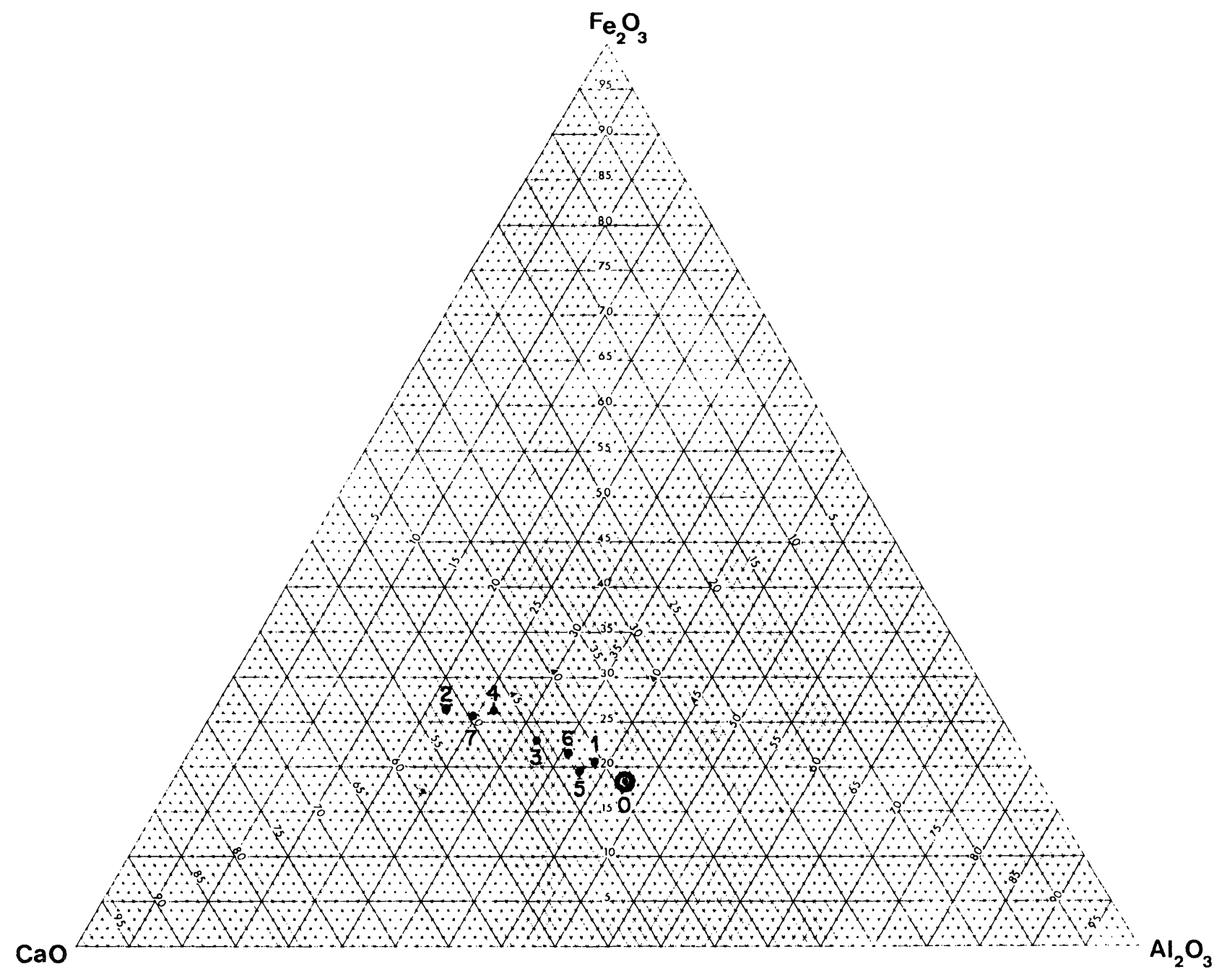

Fig.1.- Evolución de la composición absoluta de óxidos mayoritarios de los residuos insolubles del ataque alcalino.

Fig.1.- Variation of the composition of insoluble residue of alkaline attack.

0.- pasta pura hidratada/Neat paste hydrated; 1 .- $\mathrm{K}_{2} \mathrm{CO}_{3} 2 \mathrm{M} 80^{\circ} \mathrm{C} 7 \mathrm{~d} ; 2 .-\mathrm{NaOH} 2 \mathrm{M} 80^{\circ} \mathrm{C} 7 \mathrm{~d} ; 3 .-\mathrm{K}_{2} \mathrm{CO}_{3} 2 \mathrm{M} 80^{\circ} \mathrm{C} 30 \mathrm{~d} ; 4 .-\mathrm{NaOH} 2 \mathrm{M}$ $80^{\circ} 30 \mathrm{~d} ; 5 .-\mathrm{K}_{2} \mathrm{CO}_{3} 2 \mathrm{M} 20^{\circ} \mathrm{C} 30 \mathrm{~d} ; 6 .-\mathrm{K}_{2} \mathrm{CO}_{3} 2 \mathrm{M} 60^{\circ} \mathrm{C} 30 \mathrm{~d} ; 7 .-\mathrm{K}_{2} \mathrm{CO}_{3} 2 \mathrm{M} 80^{\circ} \mathrm{C} 150 \mathrm{~d}$.

\section{Efecto debido al ataque alcalino:}

Serie E: Baja dosificación con tratamiento alcalino a $80^{\circ} \mathrm{C}, 30$ días y $\mathrm{K}_{2} \mathrm{CO}_{3} 2 \mathrm{M}$.

Serie $\mathrm{F}$ : Alta dosificación con tratamiento alcalino a $80{ }^{\circ} \mathrm{C}, 30$ días y $\mathrm{K}_{2} \mathrm{CO}_{3} 2 \mathrm{M}$.

Serie G: Alta dosificación con tratamiento alcalino a $80^{\circ} \mathrm{C}, 30$ días y $\mathrm{NaOH} 2 \mathrm{M}$.

Serie H: Baja dosificación con tratamiento alcalino a $80^{\circ} \mathrm{C}$ y $\mathrm{NaOH} 2 \mathrm{M}$.

Serie I: Alta dosificación con tratamiento alcalino a $80{ }^{\circ} \mathrm{C}$ y $\mathrm{NaOH} 0,5 \mathrm{M}$.

Serie K: Baja dosificación con tratamiento térmico a $80{ }^{\circ} \mathrm{C}$ y $\mathrm{NaOH} 0,5 \mathrm{M}$.

Los resultados se exponen en la Tabla V.

\section{Effect due to the alkaline attack}

Series E: Low dosage with alkaline treatment at $80^{\circ} \mathrm{C}$, 30 days and $\mathrm{K}_{2} \mathrm{CO}_{3} 2 \mathrm{M}$

Series F: High dosage with alkaline treatment at $80^{\circ} \mathrm{C}$, 30 days and $\mathrm{K}_{2} \mathrm{CO}_{3} 2 \mathrm{M}$

Series G: High dosage with alkaline treatment at $80^{\circ} \mathrm{C}$, 30 days and $\mathrm{NaOH} 2 \mathrm{M}$

Series $H:$ Low dosage with alkaline treatment at $80^{\circ} \mathrm{C}$ and $\mathrm{NaOH} 2 \mathrm{M}$

Series I:High dosage with alkaline treatment at $80^{\circ} \mathrm{C}$ and $\mathrm{NaOH} 0.5 \mathrm{M}$.

Series K:Low dosage with alkaline treatment at $80^{\circ} \mathrm{C}$ and $\mathrm{NaOH} 0.5 \mathrm{M}$

The results are shown in Table $V$. 
Las disoluciones de ataque de las micro-probetas han sido analizadas en su contenido de aluminato $\mathrm{Al}(\mathrm{OH})_{4}^{-}$. Los resultados están expuestos en la Tabla VIII.

\subsubsection{Morteros Rilem}

En los morteros Rilem todas las series de alta dosificación son de $470 \mathrm{~kg} / \mathrm{m}^{3}$ (Tabla IX).

\section{Efecto debido a la conversión:}

Serie L: Controles.

Serie M: Con tratamiento únicamente térmico a $80^{\circ} \mathrm{C}$.

\section{Efecto debido al tratamiento alcalino:}

Serie N: Con tratamiento alcalino a $8^{\circ} \mathrm{C}, 120$ días y $\mathrm{K}_{2} \mathrm{CO}_{3} 2 \mathrm{M}$.

Serie O: Con tratamiento alcalino a $8^{\circ} \mathrm{C}, 120$ días y $\mathrm{Na}_{2} \mathrm{CO}_{3}$ saturada.

Serie $\mathrm{P}$ : Con tratamiento alcalino a $8^{\circ} \mathrm{C}, 120$ días y $\mathrm{NaOH} 2 \mathrm{M}$.

Los resultados están expuestos en las Tablas VI y VII.
The attack solutions of the micro-samples were analyzed for their contents of aluminate $\left[\mathrm{Al}(\mathrm{OH})_{4}\right]^{-}$. The results are given in Table VIII:

\subsubsection{Rilem mortars}

With the Rilem mortars all the high dosage series are of $470 \mathrm{~kg} / \mathrm{m}^{3}$ (Table LX).

\section{Effect due to the conversion}

\section{Series L:Controls}

Series M: With solely heat treatment at $80^{\circ} \mathrm{C}$.

\section{Effect due to the alkaline treatment}

Series $N$ : With alkaline treatment at $8^{\circ} \mathrm{C}, 120$ days and $\mathrm{K}_{2} \mathrm{CO}_{3} 2 \mathrm{M}$.

Series $O$ : With alkaline treatment at $8^{\circ} \mathrm{C}, 120$ days and $\mathrm{Na}_{2} \mathrm{CO}_{3}$ saturated.

Series $P$ : With alkaline treatment at $8^{\circ} \mathrm{C}, 120$ days and $\mathrm{NaOH} 2 \mathrm{M}$.

The results are shown in Tables VI and VII.

TABLA IV (TABLE IV)

Efecto de la temperatura sobre las resistencias a la compresión (Effect of the temperature on the compressive strengths)

Micro - Ensayos / Micro - Probes

\begin{tabular}{|c|c|c|c|c|c|}
\hline \multicolumn{6}{|c|}{ 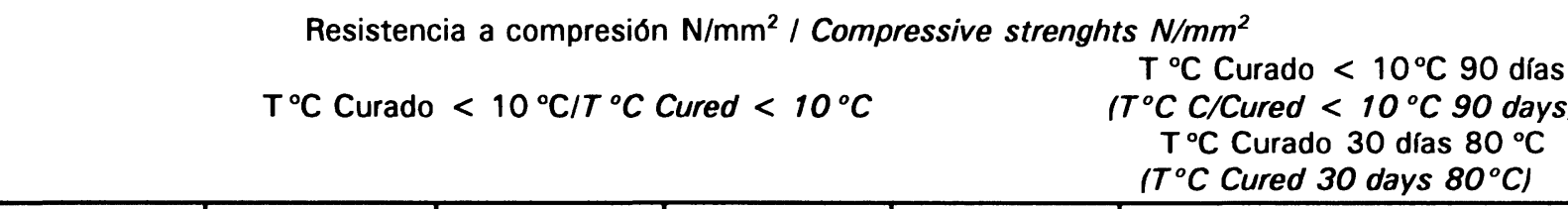 } \\
\hline$R 7 \mathrm{~d}$ & R $7 d$ & R 28 d & R $90 \mathrm{~d}$ & R 120 d & R 120 d \\
\hline $\begin{array}{c}\text { Serie A baja } \\
\text { (Serie A low) } \\
n^{\circ} \text { Tests } \\
\text { Xi } \\
\sigma \\
\text { C.V. } \%\end{array}$ & $\begin{array}{c}\text { Dosificación } \\
\text { (Dosage) } \\
4 \\
20,3 \\
2,5 \\
12,2\end{array}$ & $\begin{array}{c}8 \\
40,0 \\
8,8 \\
14,5\end{array}$ & $\begin{array}{c}6 \\
38,0 \\
4,0 \\
10,5\end{array}$ & $\begin{array}{c}11 \\
36,7 \\
2,7 \\
7,4\end{array}$ & $\begin{array}{c}\text { Serie C baja dosificación } \\
\text { (Serie C low dosage) } \\
13 \\
36,0 \\
6,9 \\
18,8\end{array}$ \\
\hline $\begin{array}{c}\text { Serie B alta } \\
\text { (Serie B high) } \\
n^{\circ} \text { Tests } \\
\text { Xi } \\
\sigma \\
\text { C.V. } \%\end{array}$ & $\begin{array}{c}\text { Dosificación } \\
\text { (Dosage) } \\
4 \\
30,0 \\
5,1 \\
17,0\end{array}$ & $\begin{array}{c}12 \\
55,1 \\
9,2 \\
16,7\end{array}$ & $\begin{array}{c}5 \\
53,0 \\
9,5 \\
17,9\end{array}$ & $\begin{array}{c}15 \\
51,7 \\
8,3 \\
16,0\end{array}$ & $\begin{array}{c}\text { Serie D alta dosificación } \\
\text { (Serie D high dosage) } \\
14 \\
48,1 \\
6,6 \\
13,8\end{array}$ \\
\hline
\end{tabular}


Efecto de la acción alcalina sobre las resistencias a la compresión (Effect of the alkaline action on the compressive strengths)

Micro - Ensayos (Micro - Probes)

Resistencia a la compresión $\mathrm{N} / \mathrm{mm}^{2}$

\begin{tabular}{|c|c|c|c|}
\hline \multicolumn{4}{|c|}{$\begin{aligned} & \mathrm{T}^{\circ} \mathrm{C} \text { Curado inmersión }\left(<10^{\circ} \mathrm{C}>\right.\left.+2^{\circ} \mathrm{C}\right) 90 \text { dias }+\mathrm{T}^{\circ} \mathrm{C} \text { Curado inmersión } 30 \text { días } 80^{\circ} \mathrm{C}=120 \mathrm{~d} \\
&\left(\text { Compressive strenghts } \mathrm{N} / \mathrm{mm}^{2}\right) \\
&\left(\mathrm{T}^{\circ} \mathrm{C} \text { Cured immersion }\left(<10^{\circ} \mathrm{C}>+2^{\circ} \mathrm{C}\right) 90 \text { days }+\mathrm{T}^{\circ} \mathrm{C} \text { Cured immersion } 30 \text { days } 80^{\circ} \mathrm{C}=120 \mathrm{~d}\right)\end{aligned}$} \\
\hline & $\mathrm{K}_{2} \mathrm{CO}_{3} 2 \mathrm{M}$ & $\mathrm{NaOH} 2 \mathrm{M}$ & $\mathrm{Na} \mathrm{OH} 0,52 \mathrm{M}$ \\
\hline $\begin{array}{c}\text { Dosificación } \\
\text { (Dosage) } \\
\mathrm{n}^{\circ} \text { Tests } \\
\mathrm{Xi} \\
\sigma \\
\text { C.V. \% }\end{array}$ & $\begin{array}{c}\text { Serie E baja } \\
\text { (Serie E low) } \\
8 \\
12,9 \\
1,7 \\
13,1\end{array}$ & $\begin{array}{c}\text { Serie H baja } \\
\text { (Serie } H \text { low) } \\
11 \\
6,0 \\
5,7 \\
9,5\end{array}$ & $\begin{array}{c}\text { Serie K baja } \\
\text { (Serie } K \text { low) } \\
10 \\
13,0 \\
9,1 \\
11,2\end{array}$ \\
\hline $\begin{array}{c}\text { Dosificación } \\
\text { (Dosage) } \\
\mathrm{n}^{\circ} \text { Tests } \\
\mathrm{Xi} \\
\sigma \\
\text { C.V. \% }\end{array}$ & $\begin{array}{c}\text { Serie F alta } \\
\text { (Serie F high) } \\
8 \\
28,4 \\
4,4 \\
15,3\end{array}$ & $\begin{array}{c}\text { Serie G alta } \\
\text { (Serie G high) } \\
8 \\
21,0 \\
3,4 \\
16,1\end{array}$ & $\begin{array}{c}\text { Serie I alta } \\
\text { (Serie I high) } \\
15 \\
39,2 \\
5,2 \\
13,2\end{array}$ \\
\hline
\end{tabular}

TABLA VI (TABLE VI)

Efecto de la temperatura sobre las resistencias a compresión (Effect of the temperature on the compressive strengths)

Ensayos Rilem (Rilem probes)

\begin{tabular}{|c|c|c|c|c|}
\hline \multicolumn{5}{|c|}{$\begin{array}{c}\text { Resistencia a la compresión } \mathrm{N} / \mathrm{mm}^{2} \\
\left(\mathrm{~T}{ }^{\circ} \mathrm{C} \text { Curado inmersion }\left(<10^{\circ} \mathrm{C}>+2^{\circ} \mathrm{C}\right) 410 \text { dias }\left(+ \text { Curado } 30 \text { dias a } 80^{\circ} \mathrm{C}\right)\right. \\
\left(\text { Compressive strengths } \mathrm{N} / \mathrm{mm}^{2}\right) \\
\left(T^{\circ} \mathrm{C} \text { Cured immersion }\left(<10^{\circ} \mathrm{C}>+2^{\circ} \mathrm{C}\right) 410 \text { days }\left(+ \text { Cured } 30 \text { days a } 80^{\circ} \mathrm{C}\right)\right.\end{array}$} \\
\hline & $R 28 d$ & R $290 d$ & R $410 \mathrm{~d}$ & R $410 d$ \\
\hline $\begin{array}{c}\text { Baja dosificación } \\
\text { (Dosage low) }\end{array}$ & & & & $\begin{array}{l}\text { Alta dosificación } \\
\text { (Dosage high) }\end{array}$ \\
\hline $\begin{array}{c}\text { Serie L } \\
\mathrm{n}^{0} \text { Tests } \\
\mathrm{Xi} \\
\sigma \\
\text { C.V. \% }\end{array}$ & $\begin{array}{c}4 \\
82,7 \\
2,0 \\
2,4\end{array}$ & $\begin{array}{l}9 \\
102,5 \\
9,5 \\
9,3\end{array}$ & $\begin{array}{c}7 \\
100,1 \\
7,3 \\
7,3\end{array}$ & $\begin{array}{c}\text { Serie M } \\
12 \\
68,9 \\
9,7 \\
14,1\end{array}$ \\
\hline
\end{tabular}

Las constantes físicas de todos los morteros preparados han sido determinadas según la Norma ASTM designación C-642, sustituyendo el tratamiento de ebullición por la extracción de aire al vacio en frío, así evitamos la interferencia de la temperatura al aumentar el grado de conversión. Los valores estadísticos de cada serie se dan en la Tabla II, en la que se ven las porosidades normales y altas, buscadas para que los resiiltados sean comparables con los morteros intersticiales de las vigas utilizadas en la construcción.
The physical constants of all the mortars prepared were determined in accordance with the ASTM Standard C-642, substituting the boiling treatment by vacuum air extraction when cold. We thus avoid the interference with the temperature on increasing the degree of conversion. The statistical values of each series are shown in Table II, in which can be seen the normal and high porosities, sought so that the results are comparable with the interstitial mortars of the beams used in the construction. 
Efecto de la acción alcalina sobre las resistencias a compresión (Effect of the alkaline action on the compressive strengths)

Ensayos Rilem (Rilem probes)

\begin{tabular}{|c|c|c|c|}
\hline \multicolumn{4}{|c|}{$\begin{array}{c}\text { Resistencia a la compresión } \mathrm{N} / \mathrm{mm}^{2} \\
\mathrm{~T}^{\circ} \mathrm{C} \text { Curado inmersión }\left(<10^{\circ} \mathrm{C}>+2^{\circ} \mathrm{C}\right) 320 \text { días }\left(+\mathrm{Curado} 90 \text { días }<10^{\circ} \mathrm{C},>+2{ }^{\circ} \mathrm{C}\right) \\
\left(\text { Compressive strengths } \mathrm{N} / \mathrm{mm}^{2}\right) \\
\left(\mathrm{T}^{\circ} \mathrm{C} \text { Curado immersion }\left(<10^{\circ} \mathrm{C}>2{ }^{\circ} \mathrm{C}\right) 320 \text { days }\left(+\mathrm{Cured} 90 \text { days }<10^{\circ} \mathrm{C},>+2^{\circ} \mathrm{C}\right)\right.\end{array}$} \\
\hline & R $410 \mathrm{~d}$ & R $410 \mathrm{~d}$ & R $410 \mathrm{~d}$ \\
\hline & $\mathrm{K}_{2} \mathrm{CO}_{3} 2 \mathrm{M}$ & $\mathrm{Na}_{2} \mathrm{CO}_{3}$ sat. & $\mathrm{NaOH} 2 \mathrm{M}$ \\
\hline $\begin{array}{c}\text { Alta dosificación } \\
\text { (Dosage high) } \\
\mathrm{n}^{\circ} \text { Tests } \\
\mathrm{Xi} \\
\sigma \\
\text { C.V. } \%\end{array}$ & $\begin{array}{c}\text { Serie N } \\
13 \\
76,0 \\
15,7 \\
20,6\end{array}$ & $\begin{array}{c}\text { Serie } 0 \\
\qquad \\
9 \\
68,1 \\
9,5 \\
9,3\end{array}$ & $\begin{array}{c}\text { Serie } P \\
\\
12 \\
64,3 \\
9,7 \\
15,1\end{array}$ \\
\hline
\end{tabular}

TABLA VIII (TABLE VIII)

Concentracón de aluminato en las soluciones alcalinas de curado de las micro probetas (Concentration of aluminate in the cured alkaline solutions of the micro probes)

\begin{tabular}{|c|c|c|c|}
\hline $\begin{array}{l}\text { Solución alcalina de curado } \\
30 \text { días en equilibrio } 80^{\circ} \mathrm{C} \\
\text { (Cured alkaline solution) } \\
\left(30 \text { days equilibrium } 80^{\circ} \mathrm{C}\right)\end{array}$ & $\mathrm{K}_{2} \mathrm{CO}_{3} 2 \mathrm{M}$ & $\mathrm{NaOH} 2 \mathrm{M}$ & $\mathrm{Na} \mathrm{OH} 0,5 \mathrm{M}$ \\
\hline $\begin{array}{c}\text { Series alta dosificación } \\
\text { (Series of high dosage) } \\
\mathrm{Al}(\mathrm{OH})_{4}-\mathrm{g} / \mathrm{L} \\
\end{array}$ & 54,4 & 63,1 & 19,6 \\
\hline $\begin{array}{c}\text { Series baja dosificación } \\
\text { (Series of low dosage) } \\
\mathrm{Al}(\mathrm{OH})_{4}-\mathrm{g} / \mathrm{L}\end{array}$ & 54,3 & 52,8 & 17,0 \\
\hline
\end{tabular}

\section{INFORMACIÓN DE LOS CAMBIOS DE FASES POR A.T.D., T.G. y X.R.D.}

\subsection{A.T.D. y T.G.}

Se han realizado los diagramas de Análisis Térmico Diferencial de las muestras representativas de las pastas puras y de las series de las probetas tratadas. El aparato ha sido un Netzsch Gerätebau. Los diagramas se han

\section{INFORMATION ON THE CHANGES OF PHASES BY D.T.A., G.T. and X.R.D.}

\subsection{D.T.A. and G.T.}

The diagrams of Differential Thermal Analysis of the representative samples of the neat pastes and of the series of the test pieces treated were attained. The apparatus used was a Netzsch Gerätebau. The 
Reducción de las resistencias a compresión debida al efecto de la temperatura y debida al efecto de la alcalinidad

(Reduction of compressive strengths due to the effect of the temperature and due to the effect of the alkalinity)

\begin{tabular}{|c|c|c|c|c|}
\hline \multicolumn{5}{|c|}{ Ensayos de Micro-Probetas (Tests of Micro-Probes) } \\
\hline$-\Delta \mathrm{N} / \mathrm{mm}^{2}$ & $\begin{array}{l}\text { Temperatura } 80^{\circ} \mathrm{C} \\
\text { (Temperature } 80^{\circ} \mathrm{C} \text { ) }\end{array}$ & $\mathrm{K}_{2} \mathrm{CO}_{3} 2 \mathrm{M}$ & $\mathrm{NaOH} 2 \mathrm{M}$ & $\mathrm{NaOH} 0,5 \mathrm{M}$ \\
\hline $\begin{array}{l}\text { Baja dosificación } \\
\text { (Low dosage) }\end{array}$ & $-0,7$ & $-23,8$ & $-30,7$ & $-23,7$ \\
\hline $\begin{array}{l}\text { Alta dosificación } \\
\text { (High dosage) }\end{array}$ & $-3,6$ & $-23,3$ & $-30,7$ & $-12,5$ \\
\hline \multicolumn{5}{|c|}{ Ensayos Rilem (Tetst Rilem Mortars) } \\
\hline$-\Delta \mathrm{N} / \mathrm{mm}^{2}$ & $\begin{array}{l}\text { Temperatura } 80^{\circ} \mathrm{C} \\
\text { (Temperature } 80^{\circ} \mathrm{C} \text { ) }\end{array}$ & $\mathrm{K}_{2} \mathrm{CO}_{3} 2 \mathrm{M}$ & $\mathrm{Na}_{2} \mathrm{CO}_{3} 1,2 \mathrm{M}$ & $\mathrm{NaOH} 2 \mathrm{M}$ \\
\hline $\begin{array}{c}\text { Dosificación } \\
\text { (Dosage) } \\
470 \mathrm{~kg} / \mathrm{m}^{3}\end{array}$ & $-3,2$ & $-24,1$ & $-32,0$ & $-35,8$ \\
\hline
\end{tabular}

obtenido con una razón de calentamiento de $10^{\circ} \mathrm{C} / \mathrm{min}$. La finura del material utilizado es de $88 \mu \mathrm{m}$, en una atmósfera de aire. Las muestras han sido deshidratadas previamente en equilibrio con una atmósfera de humedad relativa del $45 \%$.

Se han determinado por T.G. Análisis Térmico Gravimétrico la evolución cuantitativa de las fases encontradas por A.T.D., en la medida de lo posible. Los resultados están expuestos en la Fig. 2.

\subsection{X.R.D.}

Los resultados de difracción de rayos $\mathrm{X}$ de las pastas puras hidratadas y de los morteros estudiados, han estado realizados en un aparato Siemens D-500, equipado con monocromador de grafito. Se ha utilizado la radiación $\mathrm{K} \alpha$ del $\mathrm{Cu}$. Los resultados pueden verse en la Fig. 3.

\section{DISCUSIÓN DE RESULTADOS}

\subsection{Composición Química de la Pasta Pura Atacada}

La composición química de las pastas puras atacadas con diferentes disoluciones alcalinas se altera pasando diagrams were obtained with a heating rate of $10^{\circ} \mathrm{C} / \mathrm{min}$. The fineness of the material used was $88 \mu \mathrm{m}$ in an atmosphere of air. The samples were previously dehydrated in equilibrium with an atmosphere with a relative humidity of $45 \%$

The quantitative evolution of the phases discovered by D.T.A. was determined by G.T. Gravimetric Thermal Analysis as far as possible. The results are shown in Fig. 2.

\subsection{X.R.D.}

The results of $x$-ray diffraction of the hydrated neat pastes and of the mortars studied were obtained in a Siemens D-500 apparatus equipped with a graphite monochromator. The radiation $K \alpha$ of the $C u$ was used. The results are shown in Fig. 3.

\section{DISCUSSION OF RESULTS}

\subsection{Chemical Composition of the Neat Paste Attacked}

The chemical composition of the neat pastes attacked with different alkaline solutions is altered by passing 
aluminato a la disolución. Los valores de las concentraciones de las diferentes disoluciones son relativamente próximas, entre los 15 y $18 \mathrm{~g} / 1$, calculados como $\mathrm{Al}(\mathrm{OH})_{4}^{-}$.

Los análisis químicos de las disoluciones de ataque en el curado a $80^{\circ} \mathrm{C}$ de las series de microprobetas revelan, que la concentración de aluminio soluble depende de la concentración de iones $\mathrm{OH}$, y no depende de la masa del material sólido existente.

Por otro lado, el hecho de que los ensayos puestos en el equilibrio a la temperatura de $80^{\circ} \mathrm{C}$ durante un periódo de tiempo largo de 150 días, se mantenga el valor sobre aluminate into the solution. The values of the concentrations of the different solutions are relatively similar, between 15 and $18 \mathrm{~g} / \mathrm{l}$, calculated as $\left[\mathrm{Al}(\mathrm{OH})_{4}\right]^{-}$

The chemical analyses of the attack solutions in the curing of the series of microsamples at $80^{\circ} \mathrm{C}$ reveal that the concentration of soluble aluminate depends on the concentration of $\mathrm{OH}^{-}$ions, and does not depend on the mass of the solid material existing.

On the other hand, the fact that the tests placed in equilibrium at a temperature of $80^{\circ} \mathrm{C}$ for a period of time lasting 150 days maintain the value of $15 \mathrm{~g} / \mathrm{l}$

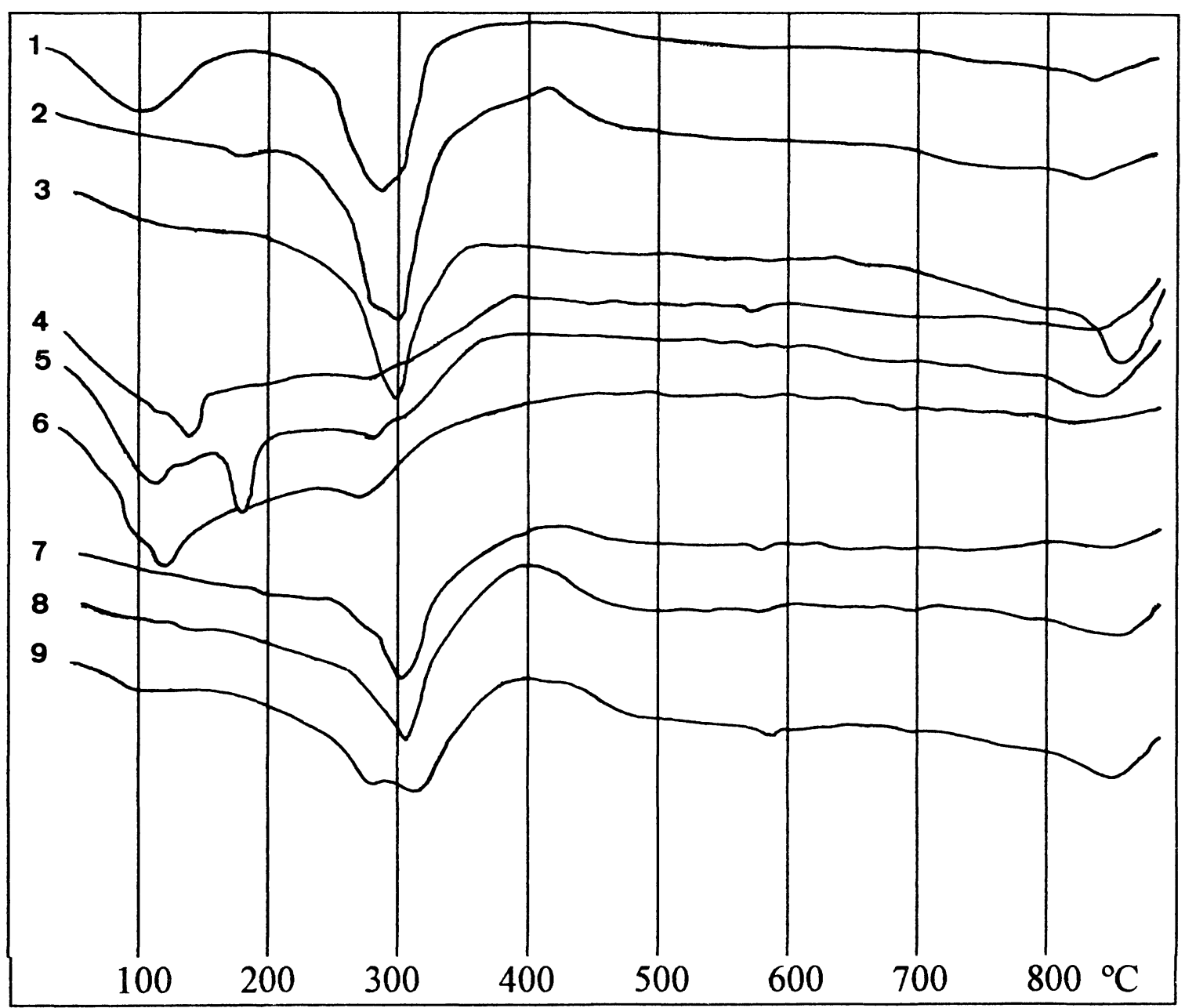

Fig.2.- Diagramas de análisis térmico diferencial

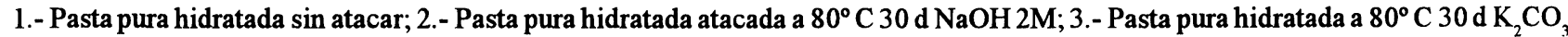
2M; 4.- Probetas Rilem L curadas $\mathrm{T}^{\mathrm{a}}<10^{\circ} \mathrm{C} 290 \mathrm{~d}$ Cuarzo; 5.- Probetas Rilem L curadas $\mathrm{T}^{\mathrm{a}}<10^{\circ} \mathrm{C} 290 \mathrm{~d}$ Caliza; 6.- Micro - probetas $B$ curadas $\mathrm{T}^{\mathrm{a}}<10^{\circ} \mathrm{C} 28 \mathrm{~d} ; 7$.- Micro - probetas $\mathrm{M}$ curadas $\mathrm{T}^{\mathrm{a}}<10^{\circ} \mathrm{C}+30 \mathrm{~d}$ trat. a $80^{\circ} \mathrm{C}$; 8.- Micro - probetas $\mathrm{F}$ curadas $\mathrm{T}^{\mathrm{a}}<10^{\circ} \mathrm{C}$ $+30 \mathrm{~d}$ trat. a $80^{\circ} \mathrm{C} \mathrm{NaOH} 2 \mathrm{M}$; 9.- Micro - probetas $\mathrm{G}$ curadas $\mathrm{T}^{\mathrm{a}}<10^{\circ} \mathrm{C}+30 \mathrm{~d}$ trat. a $80^{\circ} \mathrm{C} \mathrm{K}_{2} \mathrm{CO}_{3} 2 \mathrm{M}$

Fig.2.- DTA Curves.

1.- Neat paste pure hidrated without attack; 2.- Neat paste pure hydrated attacked a $80^{\circ} \mathrm{C} 30 \mathrm{~d} \mathrm{NaOH} 2 \mathrm{M} ; 3 .-$ Neat paste pure hydrated attacked $80^{\circ} \mathrm{C} 30 \mathrm{~d} \mathrm{~K} \mathrm{CO}_{3} 2 \mathrm{M}$;4.- Probes Rilem L cured $T^{a} \div 10^{\circ} \mathrm{C} 290 \mathrm{~d}$ Quartz; 5. - Probes Rilem L cured $T^{a}<10^{\circ} \mathrm{C} 290 \mathrm{~d}$ Limestone; 6.- Micro - probes B cured $T^{a}<10^{\circ} \mathrm{C} 28 \mathrm{~d}$; 7.- Micro - probes M curadas $T^{a} 10^{\circ} \mathrm{C}+30 \mathrm{~d}$ a $80^{\circ} \mathrm{C}$; 8.- Micro - probes F cured $\mathrm{T}^{\circ}<10^{\circ} \mathrm{C}+30 \mathrm{~d}$. a $80^{\circ} \mathrm{C} \mathrm{NaOH} 2 \mathrm{M}$; 9.- Micro - probes G cured $\mathrm{T}^{a}-10^{\circ} \mathrm{C}+30 \mathrm{~d}$ a $80^{\circ} \mathrm{C} \mathrm{K}_{2} \mathrm{CO}_{3} 2 \mathrm{M}$ 

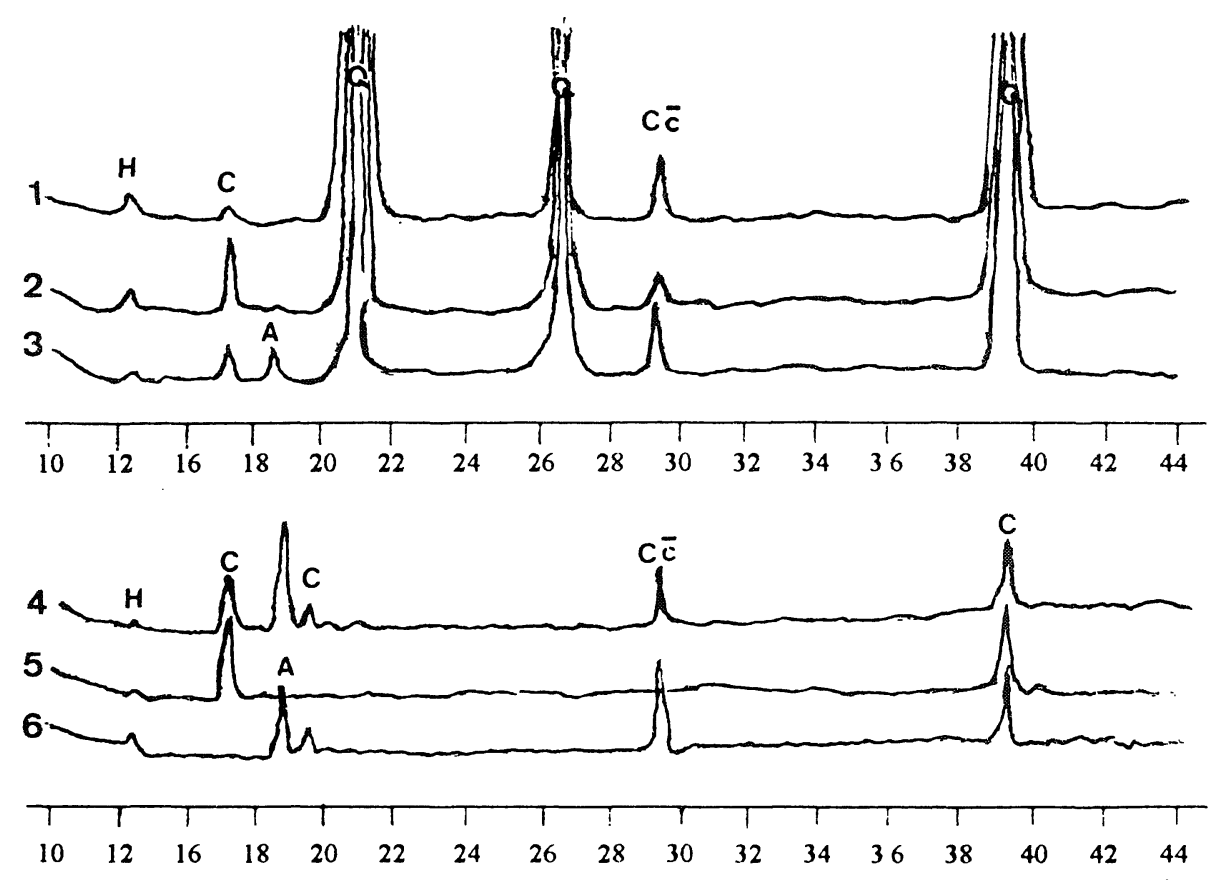

Fig.3.- XRD

1.- Micro - probetas $\mathrm{E}$ atacadas $\mathrm{K}_{2} \mathrm{CO}_{3} 2 \mathrm{M} 30 \mathrm{~d} .80^{\circ} \mathrm{C}$; 2.- Rilem tests $\mathrm{N}$ atacadas $\mathrm{NaOH} 2 \mathrm{M} 30 \mathrm{~d}$. $80^{\circ} \mathrm{C}$; 3.- Rilem tests $\mathrm{P}$ atacadas $\mathrm{K}_{2} \mathrm{CO}_{3} 2 \mathrm{M} 30 \mathrm{~d} .80^{\circ} \mathrm{C}$; 4.- Pasta pura hidratada sin ataque; 5.- Pasta pura atacada con $\mathrm{NaOH} 2 \mathrm{M} 30 \mathrm{~d}$. $80^{\circ} \mathrm{C}$; 6.- Pasta pura hidratada atacada con $\mathrm{K}_{2} \mathrm{CO}_{3} 2 \mathrm{M} 30 \mathrm{~d} .80^{\circ} \mathrm{C}$

Fig.3.- XRD

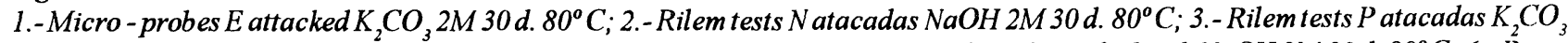
$2 \mathrm{M} 30 \mathrm{~d} .80^{\circ} \mathrm{C}$; 4. - Neat paste pure hydrated without attack; 5.- Neat paste pure hydrated attacked with $\mathrm{NaOH} 2 \mathrm{M} 30 \mathrm{~d} .80^{\circ} \mathrm{C} ; 6 .-\mathrm{Pasta}$ pura hidratada atacada con $\mathrm{K}_{2} \mathrm{CO}_{3} 2 \mathrm{M} 30 \mathrm{~d} .80^{\circ} \mathrm{C}$

un $15 \mathrm{~g} / 1$, nos confirma que se establece un equilibrio de reacción al alcanzar las condiciones de estado estacionario.

El efecto de la temperatura no es sensiblemente importante. E1 hecho de estudiar temperaturas tan altas como $80^{\circ} \mathrm{C}$, es para cubrir el límite máximo de temperaturas posibles en edificios de zonas de clima cálido.

Para análisis térmicos, se constata que las disoluciones de carbonato alcalino introducen carboaluminatos en cantidades importantes, pero, en ningún caso, desaparece la fase cúbica en las condiciones estudiadas. Este hecho enmascara la cantidad real del residuo insoluble que se obtiene. La composición de este residuo atacado acusa un notable descenso del contenido de alumina. Las cantidades de carboaluminatos introducidas modifican los valores de las pérdidas por calcinación y, en consecuencia, las cantidades de materia fija de los análisis. Este hecho obliga a considerar las masas absolutas de los componentes mayoritarios en un diagrama ternario, para poder conocer el verdadero valor de la pérdida de óxido de alúmina de la pasta atacada. confirms that an equilibrium of reaction is established on reaching the steady state conditions.

The effect of the temperature is not markedly important. The fact that we study temperatures as high as $80^{\circ} \mathrm{C}$ is to cover the maximum limit of temperatures possible in buildings in areas with a hot climate.

For thermal analyses it is shown that the solutions of alkali carbonate introduce carboaluminates in important quantities, but in no case does the cubic phase disappear under the conditions studied. This fact masks the true quantity of insoluble residue which is obtained. The composition of this attacked residue reveals a notable decrease in the contents of alumina. The quantities of carboaluminates introduced modify the values of the losses on ignition and, consequently, the quantities of fixed material of the analyses. This fact makes it necessary to consider the absolute masses of the majority components in a ternary diagram in order to find out the true value of the loss of alumina oxide of the tracted paste by alkaline attack. 
El valor llega a un máximo de un $20 \%$ en un tiempo de ataque de 150 días sobre la masa absoluta de mayoritarios $\mathrm{CaO}, \mathrm{Al}_{2} \mathrm{O}_{3}$ y $\mathrm{Fe}_{2} \mathrm{O}_{3}$, cuando la disolución es de carbonato potásico. Con hidróxido sódico, un valor próximo se obtiene en 7 días de contacto a $80^{\circ} \mathrm{C}$.

\subsection{Influencia de la Temperatura sobre las Resistencias}

El efecto de la temperatura en los ensayos se ha encontrado, inesperadamente, muy bajo (Tabla IV), de unos 0,7 a $3,6 \mathrm{~N} / \mathrm{mm}^{2}$ respectivamente, para las series de alta y baja dosificación. Esto es debido, probablemente, a que las microprobetas del conjunto, y que han estado conservadas a temperatura inferior a $10^{\circ} \mathrm{C}$, el grado de conversión de las probetas control era ya considerable a los 120 días, de acuerdo con la información obtenida por ATD, (Fig. 2).

Este hecho, en comparación con los ensayos Rilem, con más alta dosificación y menor porosidad, nos revela que la relación gel/poros tiene una influencia en la valocidad de convsersión.

\subsection{Influencia del Ataque Alcalino sobre las Resistencias}

Para las series de micro-probetas de baja dosificación, el ataque alcalino produce una bajada de resistencia considerable, evaluada en $23 \mathrm{~N} / \mathrm{mm}^{2}$ para $\mathrm{NaOH} 2 \mathrm{M}$.

En el caso de los ensayos Rilem, de forma similar, la disminución de las resistencias se encuentra con $\mathrm{NaOH}$ $2 \mathrm{M}$ y su valor registrado, es el más alto. Le sigue el ataque con carbonato sódico $1,2 \mathrm{M}$, equivalente a la saturación a unos $10^{\circ} \mathrm{C}$ de acuerdo con las condiciones de ataque, y el carbonato potásico $2 \mathrm{M}$.

Teniendo en cuenta que la influencia de la temperatura se superpone con el ataque alcalino, los efectos de la temperatura se han de descontar de la disminución total obtenida por el ataque a $80^{\circ} \mathrm{C}$.

Si consideramos las resistencias residuales, en lugar de incremento negativo en el resultado del ataque alcalino, observamos que las dosificaciones bajas dan lugar a resistencias residuales especialmente bajas. Esto se justifica por la porosidad elevada que tienen estos morteros, y en consecuencia, la relación "gel/poro" del material es también baja.

Este parámetro es decisivo para comprender el comportamiento del cemento aluminoso como aglomerante, porqué incide en una doble vertiente: una, la más fácil penetrabilidad de la disolución agresiva, y la otra, la más alta debilidad de las uniones físico-químicas
The value reaches a maximum of $20 \%$ in an attacking time of 150 days on the absolute mass of majority components $\mathrm{CaO}, \mathrm{Al}_{2} \mathrm{O}_{3}$ and $\mathrm{Fe}_{2} \mathrm{O}_{3}$, when the solution is potassium carbonate. With sodium hydroxide, a similar value is obtained in 7 days of contact at $80^{\circ} \mathrm{C}$.

\subsection{Influence of the Temperature on the Strengths}

The effect of the temperature on the tests was unexpectedly found to be very low, Table IV, with some 0.7 to $3.6 \mathrm{~N} / \mathrm{mm}^{2}$, respectively, for the high and low dosage series. This is probably due to the fact that, in spite of the fact that the microsamples were maintained at a temperature below $10^{\circ} \mathrm{C}$, the degree of conversion for the control tests was already high at 120 days, according to the information obtained by DTA, Fig. 2.

This fact, compared with the Rilem tests, with a higher dosage and less porosity, shows that the gel/pore ratio has a great influence on the speed of conversion.

\subsection{Influence of the Alkaline Attack on the Strengths}

With regard to the series of low dosage microsamples the alkaline attack produced a considerable drop in strength assessed at some $23 \mathrm{~N} / \mathrm{mm}^{2}$ for $\mathrm{NaOH} 2 \mathrm{M}$.

In the case of the Rilem tests the reduction of the strengths also occurs with $\mathrm{NaOH} 2 \mathrm{M}$ and its registered value is the highest. It is followed by the attack with sodium carbonate $1.2 \mathrm{M}$, equivalent to saturation at some $10^{\circ} \mathrm{C}$ in accordance with the conditions of attack, and potassium carbonate $2 \mathrm{M}$.

Taking into account that the influence of the temperature overlaps with the alkaline attack, the effects of the temperature have to be discounted from the total reduction obtained by the attack at $80^{\circ} \mathrm{C}$.

If we consider the residuary strengths, instead of a negative increase in the result of the alkaline attack, we observe that the low dosages give rise to especially low residuary strengths. This is explained by the high porosity of these mortars, and consequently the "gel/pore" ratio of the material is also low.

This parameter is decisive in order to understand the behaviour of the aluminóus cement as an agglomerant, because it affects a double aspect: firstly, the easier penetrability of the aggressive solution, and secondly the greater weakness of the physical-chemical unions of the 
de los geles en su acción aglomerante. Considerando esta situación, el equilibrio "hidratación-deshidratación" actúa negativamente sobre las resistencias y, en consecuencia, se entra en una situación de parámetros de riesgo. Cuando la humedad relativa ambiental es del $60 \%$, la cantidad de agua dentro de los poros accesibles del hormigón está alrededor de un $50 \%$, es decir, que necesitaríamos $0,40 \%$ de hidróxido sódico, por ejemplo, en el hormigón, para tener una concentración de iones $\mathrm{OH}^{-} 2 \mathrm{M}$, que es la que corresponde a los ensayos realizados en este trabajo.

\subsection{Los Cambios de Fases en los Procesos Estudiados}

Las altas cantidades de fases hidratadas $\mathrm{CAH}_{10}, \mathrm{\gamma}-\mathrm{Al}_{2} \mathrm{O}_{3}$ y $\mathrm{Al}_{2} \mathrm{O}_{3}$ gel, tanto en las pastas puras como en las probetas preparadas y curadas a baja temperatura antes de cualquier tratamiento, encontradas por $\operatorname{ATD}(1,4,5$ y 6$)$, (Fig. 2), no se revelan por XRD.

Este hecho, de acuerdo con autores precedentes, como BUSHNELL-WATSON (8), confirma que son fases amorfas y por eso no se revelan por XRD hasta que, a través de la conversión, cristalizan, y una vez cristalizadas, dan lugar a difracción de rayos $\mathrm{X}$ y pueden, entonces, ser reveladas, como sucede en los diagramas de los morteros y pastas tratadas.

Las altas resistencias del cemento aluminoso, evidencian que tienen relación con este estado de gel. A medida que se va convirtiendo, bajan. Por tanto, para evaluar las resistencias en el seguimiento técnico del estado de los hormigones de los techos, es mejor la información obtenida por ATD.

La acción del carbonato da lugar a la formación de cantidades de $\mathrm{C}_{4} \mathrm{AcH}_{11}$. Es posible que la incorporación de esta fase justifique el porqué los resultados de las resistencias de las series $\mathbf{N}$ y $\mathbf{O}$, con carbonatos alcalinos como atacantes, den menor bajada en la resistencia que las tratadas con $\mathrm{NaOH}$, serie $\mathbf{P}$.

\section{CONCLUSIONES}

-La disolución alcalina de las fases del cemento aluminoso hidratado se produce dentro de las zonas de concentración de $\mathbf{O H}-$ estudiadas, y afecta negativamente a las resistencias residuales.

Los resultados analíticos confirman que la disolución alcalina está limitada por un equilibrio químico, cuando las condiciones de contacto están estancadas. El valor del aluminato $\mathrm{Al}(\mathbf{O H})_{4}^{--}$soluble depende de la concentración de los iones $\mathbf{O H}^{-}$, y la temperatura no tiene gran influencia. gels in their bonding action. When this situation occurs, the "hydration/dehydration" equilibrium acts negatively on the strengths and, consequently, a situation of parameters of risk arises. Considering a relative ambient humidity of $60 \%$, the quantity of water inside the accessible pores of the concrete is around $50 \%$, that is to say that we would need, for example, $0.40 \%$ of sodium hydroxide in the concrete in order to have a concentration of $\mathrm{OH}^{-i o n s} 2 \mathrm{M}$, which is that which corresponds to the tests carried out in this work.

\subsection{The Changes of Phases in the Processes Studied}

The high quantities of hydrated phases $\mathrm{CAH}_{10} \gamma-\mathrm{Al}_{2} \mathrm{O}_{3}$ and $\mathrm{Al}_{2} \mathrm{O}_{3} \mathrm{gel}$, both in the neat pastes and in the test pieces prepared and cured at low temperature before any treatment, found by DTA (1, 4, 5 and 6 Fig. 2) are not revealed by $X R D$.

This fact, according to preceding authors, such as BUSHNELL-WATSON (8), confirms that they are amorphous phases and they are not therefore revealed by XRD until, through the conversion, they crystallize and, once crystallized, they give rise to $x$-ray diffraction and can then be revealed, as occurs in the diagrams of the mortars and pastes treated.

It is obvious that the high strengths of the aluminous cement are related to this gel state. As they are converted, they descend. Therefore, in order to assess the strengths in the technical monitoring of the state of the concrete of the roofs, the information obtained by DTA is better.

The action of the carbonate gives rise to the formation of important quantities of $\mathrm{C} \mathrm{AcH}_{11}$. It is possible that the incorporation of this phase explains how the results of the strengths of the $\boldsymbol{N}$ and $\boldsymbol{O}$ series, with alkaline carbonates as attacker, give a lower drop in strength than those treated with $\mathrm{NaOH}$, series $P$.

\section{CONCLUSIONS}

-The alkaline solution of the phases of the hydrated aluminous cement occurs inside the areas of concentration of $\mathrm{OH}^{-}$studied, and negatively affects the residuary strengths.

The analytical results confirm that the alkaline solution is limited by a chemical equilibrium when the contact conditions are static. The value of the soluble aluminate $\mathrm{Al}(\mathrm{OH})_{\mathrm{d}}^{-}$depends on the concentration of the $\mathrm{OH}^{-}$ions, and the temperature does not have a great influence. 
-Morteros RILEM con dosificaciones altas de cemento $470 \mathrm{~kg} / \mathrm{m}^{3}$, curados a baja temperatura en inmersión y sin convertir, experimentan una disminución de resistencias a la compresión de $38,5 \mathrm{~N} / \mathrm{mm}^{2}$, después de 30 días de ataque con una disolución de $\mathrm{NaOH} 2 \mathrm{M}$ a $80{ }^{\circ} \mathrm{C}$.

Los morteros tratados tienen una resistencia residual de $64 \mathrm{~N} / \mathrm{mm}^{2}$.

-Morteros de baja dosificación, del orden de $250 \mathrm{~kg} / \mathrm{cm}^{3}$, curados en inmersión a baja temperatura, después del ataque con disolución alcalina de $\mathrm{NaOH} 2 \mathrm{M}$ a $80^{\circ} \mathrm{C}$, experimentan resistencias residuales de $6 \mathrm{~N} / \mathrm{mm}^{2}$, las cuales podrían dar lugar una situación de riesgo por colapso.

\section{AGRADECIMIENTOS}

Este trabajo se ha realizado con el soporte del CENTRE TĖCNIC I DE COOPERACIÓ PER A LA REHABILITACIÓ D'HABITAGES DEL DEPARTAMENT DE POLÍTICA TERRITORIAL I OBRES PUBLIQUES DE LA GENERALITAT DE CATALUNYA.
-RILEM mortars with high dosages of cement, $470 \mathrm{~kg} / \mathrm{m}^{3}$, cured at low temperature by immersion and with very low conversion degree, experience a reduction in compressive strengths of $38.5 \mathrm{~N} / \mathrm{mm}^{2}$, after 30 days of attack with a solution of $\mathrm{NaOH} 2 \mathrm{M}$ at $80^{\circ} \mathrm{C}$.

The treated mortars have a residuary strength of $64 \mathrm{~N} / \mathrm{mm}^{2}$.

-Mortars with a low dosage in the order of $250 \mathrm{~kg} / \mathrm{m}^{3}$, cured by immersion at a low temperature, after the attack with an alkaline solution of $\mathrm{NaOH} 2 \mathrm{M}$ at $80^{\circ} \mathrm{C}$, experience residuary strengths of $6 \mathrm{~N} / \mathrm{mm}^{2}$, which could lead to a situation of risk of collapse.

\section{ACKNOWLEDGEMENT}

This work was undertaken with the support of the CENTRE TECNIC I DE COOPERACIÓ PER A LA REHABILITACIÓ D'HABITATGES DEL DEPARTAMENT DE POLITICA TERRITORIAL I OBRES PUBLIQUES DE LA GENERALITAT DE CATALUNYA.

\section{BIBLIOGRAFÍA}

(1) GEORGE, C.M.: "Aluminous cement". A review of recent literature 7th International Congress on the Chemistry of Cement, Vol. I, Principal Reports V-1/3 - 26. Paris 1980.

(2) MIDGLEY, H.G.: "The chemical resistance of high alumina cement concrete". 7th International Congress on the Chemistry of Cement, Vol. III, Communications V-85/87. Paris 1980.

(3) RENGADE, E. and L'HOSPITALLIER, P.: "Recherches sur les causes de certain ph'enoménes d'alteration des bétons de ciment alumineaus". Revue des materiaux de construction et de travaux publics 318, 1936.

(4) GOMÁ, F.: “Acerca de la determinació gravimètrica de la sílice”. Tesi Doctoral 1975, Universitat deBarcelona. 1975, Departament de Química Analítica.

(5) GOMÁ, F.: "Nuevo método gravimétrico para la determinación de sílice en materiales silíceos". Cemento-Hormigón, n505, marzo $1976,40 \mathrm{pp}$.

(6) GOMÁ, F.: "Generalized method Formicontrol the determination of the composition of portland cement mixtures with active additions". 8th International Congress on the Chemistry of Cement. Rio de Janeiro,1986. IV - 113/117.

(7) Analyse Chimique des Ciments CEN EN 162 - 2, 1987.

(8) BUSHNELL - WATSON, S.M.: "La aplicación del análisis térmico a las reacciones de hidratación y conversión de cementos aluminosos". Materiales de Construcción, Vol. 42, n² 228. 1992. Madrid. 